ARTICLE

https://doi.org/10.1038/s41467-018-07653-5

OPEN

\title{
CryoEM reveals how the complement membrane attack complex ruptures lipid bilayers
}

\author{
Anaïs Menny ${ }^{1}$, Marina Serna ${ }^{1,2}$, Courtney M. Boyd ${ }^{1}$, Scott Gardner ${ }^{1}$, Agnel Praveen Joseph 3,6 , B. Paul Morgan ${ }^{4}$, \\ Maya Topf (i) ${ }^{3}$, Nicholas J. Brooks (i) ${ }^{5} \&$ Doryen Bubeck (i) ${ }^{1}$
}

The membrane attack complex (MAC) is one of the immune system's first responders. Complement proteins assemble on target membranes to form pores that lyse pathogens and impact tissue homeostasis of self-cells. How MAC disrupts the membrane barrier remains unclear. Here we use electron cryo-microscopy and flicker spectroscopy to show that MAC interacts with lipid bilayers in two distinct ways. Whereas $\mathrm{C} 6$ and $\mathrm{C} 7$ associate with the outer leaflet and reduce the energy for membrane bending, C8 and C9 traverse the bilayer increasing membrane rigidity. CryoEM reconstructions reveal plasticity of the MAC pore and demonstrate how $\mathrm{C} 5 \mathrm{~b} 6$ acts as a platform, directing assembly of a giant $\beta$-barrel whose structure is supported by a glycan scaffold. Our work provides a structural basis for understanding how $\beta$-pore forming proteins breach the membrane and reveals a mechanism for how MAC kills pathogens and regulates cell functions.

\footnotetext{
${ }^{1}$ Department of Life Sciences, Sir Ernst Chain Building, Imperial College London, London SW7 2AZ, UK. ${ }^{2}$ Spanish National Cancer Research Centre, CNIO, Melchor Fernández Almagro, 3.28029 Madrid, Spain. ${ }^{3}$ Institute of Structural and Molecular Biology, Department of Biological Sciences, Birkbeck, University of London, Malet Street, London WC1E 7HX, UK. ${ }^{4}$ Division of Infection and Immunity, School of Medicine, Cardiff University, Heath Park, Cardiff CF14 4XN, UK. ${ }^{5}$ Department of Chemistry, Imperial College London, London SW7 2AZ, UK. ${ }^{6}$ Present address: Scientific Computing Department, Science and Technology Facilities Council, Research Complex at Harwell, Didcot OX11 OFA, UK. These authors contributed equally: Marina Serna, Courtney M. Boyd. Correspondence and requests for materials should be addressed to D.B. (email: d.bubeck@imperial.ac.uk)
} 
T he multiprotein membrane attack complex is a bactericidal weapon of the innate immune system that also modulates inflammation and proliferation when formed on self-cells ${ }^{1}$. The MAC pore targets a wide range of pathogens, forming on and killing Gram-negative bacteria ${ }^{2}$, enveloped viruses ${ }^{3}$, and parasites $^{4}$. This innate immune effector is essential for fighting bacteria of the genus Neisseria ${ }^{5}$ with genetic deficiencies in component proteins leading to recurrent infections ${ }^{6}$. Host cells are protected from bystander damage by the GPI-anchored receptor $\mathrm{CD} 59$, the only membrane-bound inhibitor of $\mathrm{MAC}^{7}$. Deficiency of CD59 causes the lethal blood disorder Paroxysmal Nocturnal Hemoglobinuria, a disease characterized by thrombosis and chronic hemolysis ${ }^{8}$. Complement activation and MAC formation also contributes to killing of cancer cells during immunotherapy treatments ${ }^{9}$. Therefore, developing a molecular model of how MAC forms on target cells is essential for understanding the immune response to microbes and for the development of therapeutics that regulate complement activity.

MAC assembles from soluble complement proteins in an obligate sequential pathway ${ }^{10}$. In the presence of membranes, C5b6 (a complex comprised of $\mathrm{C} 5 \mathrm{~b}$ and $\mathrm{C}^{11}{ }^{11}$ ) binds $\mathrm{C} 7$ to form the lipophilic MAC precursor $\mathrm{C} 5 \mathrm{~b} 7^{12}$. C8 irreversibly binds the nascent complex, resulting in the membrane-inserted $\mathrm{C}_{5} \mathrm{~b} 8^{13}$. C9 molecules associate with $\mathrm{C} 5 \mathrm{~b} 8$ in the membrane to form $\mathrm{C} 5 \mathrm{~b} 9$ and polymerize to complete the MAC pore ${ }^{14}$. Previous electron cryo-microscopy (cryoEM) reconstructions of the complex revealed that the final MAC is comprised of 6 polypeptide chains (C5b, C6, C7, C8a, C8 3 , and C8 $\gamma$ ) together with 18 C9 monomers that are arranged in a split-washer configuration ${ }^{15,16}$. Based on structural similarity with bacterial homologs, the giant $(110 \AA$ diameter) $\beta$-barrel pore is formed when helical bundles in the Membrane Attack Complex-Perforin (MACPF) domains transform into transmembrane $\beta$-hairpins $(\mathrm{TMH})^{17-19}$, although a molecular mechanism for how this occurs is not currently understood.

In this study, we used cryoEM to determine the structures of two MAC conformations at near atomic resolution and derive a nearly complete atomic model for the pore. In combination with flicker spectroscopy, we show how MAC assembly impacts biophysical properties of the bilayer and resolve the mechanisms of membrane interaction and MAC activity.

\section{Results}

CryoEM structure of the MAC. The human MAC pore was formed on liposomes from individual complement proteins. The lipid composition of vesicles was selected based on the stoichiometric homogeneity of deposited pores. MAC was solubilized with detergent and purified for structural studies, as described previously $^{15}$. By integrating newly collected data across multiple electron microscopes (Supplementary Table 1), we were able to improve the overall resolution of the MAC from $8.5 \AA^{15}$ to $4.9 \AA$; however, density corresponding to the interface between C6 and the terminal C9 was still poorly resolved. We used 3D classification procedures to computationally isolate two stoichiometrically identical conformations, open and closed, which varied in the extent of $\beta$-barrel closure (Fig. 1 and Supplementary Fig. 1). 2D classification of negatively stained complexes inserted into a lipid monolayer confirmed the presence of these states in a membrane environment (Supplementary Fig. 2a), in agreement with cryotomography structures of MACs in liposomes ${ }^{16}$. The maps were further subdivided into three components: an asymmetric region (C5b, C6, C7, and $\mathrm{C} 8$ ), a hinge region (C7, C8, and two $\mathrm{C} 9$ molecules), and a C9 oligomer. Using a masked refinement strategy coupled with signal subtraction ${ }^{20}$, we improved the resolution of the asymmetric regions for each conformation to
$4.7 \AA$ and $5.9 \AA$ (Fig. 1, Supplementary Figs. 1, and 2b). The hinge region of the open conformation was resolved to $4.9 \AA$ (Supplementary Figs. 1 and $2 \mathrm{~b}$ ). Masked refinement from signalsubtracted images followed by sub-volume averaging was used to resolve the averaged $\mathrm{C} 9$ monomer from the open conformation at $4.4 \AA$ (Fig. 1a, and Supplementary Figs. 1, 2b, 3c). A similar analysis of the $\mathrm{C} 9$ oligomer from the closed conformation resulted in a lower resolution map. Therefore, we focused our interpretation of C9 on density derived from the open conformation oligomer. The new maps enabled us to build an atomic model that includes the irregular and asymmetric $\beta$-barrel pore (Supplementary Table 1 and Supplementary Fig. 3). Although density is lacking for many side-chains within $\mathrm{C} 5 \mathrm{~b}$ and the lower half of the central $\beta$-barrel, we have imposed experimental restraints that justify their register in the atomic model. Crystal structures for soluble components ( $\mathrm{C} 5 \mathrm{~b} 6^{11}$ and $\mathrm{C} 8{ }^{21}$, a heterotrimeric complex consisting of $\alpha, \beta$, and $\gamma$ polypeptide chains) together with homology models for C7 and C9 were fitted into the density. Domains of these structures were first refined as rigid bodies, with disulfide bond restraints. Models were further refined restraining secondary structure and side-chain geometry to higher resolution crystallographic structures. $\beta$-strands that comprise the central barrel were initiated where side-chain density was visible and extended imposing idealized backbone geometry constraints. The trajectory for each strand is linear and the register was confirmed by correlating glycan density with the position of the modified residue in the sequence (Supplementary Fig. 4).

MAC is a flexible immune pore. We used $3 \mathrm{D}$ classification to resolve MAC conformational flexibility (Fig. 1 and Supplementary Fig. 1). The open conformation is characterized by a $30 \AA$ wide chasm that runs the length of the complex (Fig. 1b). Lipid molecules likely fill the opening on the wall of the pore, reminiscent of arc pores observed for both mammalian and bacterial $\beta$-pore forming proteins ${ }^{22,23}$. The asymmetric region juts into the lumen of the barrel like a "paddle", exaggerating the MAC's split washer shape (Fig. $1 \mathrm{~b}$ and Supplementary Movie 1). C8 $\gamma$ is wedged in the crease between the rotated asymmetric component and adjacent C9 oligomer, and may limit the rotation of the paddle. While curvature of $\mathrm{C} 9$ arcs vary at either end, the central section is near-circular with monomers equally spaced $\sim 16^{\circ}$ apart (Fig. 1b, c), reminiscent of the arrangement observed for a C9 homo-oligomer ${ }^{24}$. Although the chasm is sealed in the closed conformation (Fig. 1c), interfaces mediating the MACPF-rim and transmembrane regions are not flush. The asymmetric region swings back and meets $\mathrm{C} 9$ in a noncanonical MAPCFthrombospondin (C6-TSP3) interaction with limited buried surface area (Fig. 1d). Despite a contiguous extracellular $\beta$-barrel, there remains a gap within the transmembrane pore where the shorter hairpins of $\mathrm{C} 6, \mathrm{C} 7$, and $\mathrm{C} 8$ abut those of $\mathrm{C} 9$ (Fig. 1c). In the open conformation, the first and terminal C9 are latterly shifted by $\sim 20 \AA$, while the ring of the closed conformation remains planar (Fig. 1). Therefore, conformational flexibility of the assembly may impact local curvature of the membrane.

The MAC is a highly-glycosylated assembly with all complement components post-translationally modified ${ }^{11,25-27}$. We observe density for many of the reported glycans on $\mathrm{C}^{25}$, $\mathrm{C}^{27}$, and $\mathrm{C}^{26}$, which line the $\beta$-barrel's concave face (Figs. $1 \mathrm{~b}, \mathrm{c}$, $2 \mathrm{a}$ and Supplementary Fig. 4). Glycan removal led to irregular pores with significantly distorted curvature (Supplementary Fig. 4). These data suggest glycans could play a role in maintaining the structural integrity of a flexible giant $\beta$-barrel. Although deglycosylation did not impact the ability to rupture simple model membranes (Supplementary Fig. 4), glycans may confer greater robustness on pore assembly. 
a
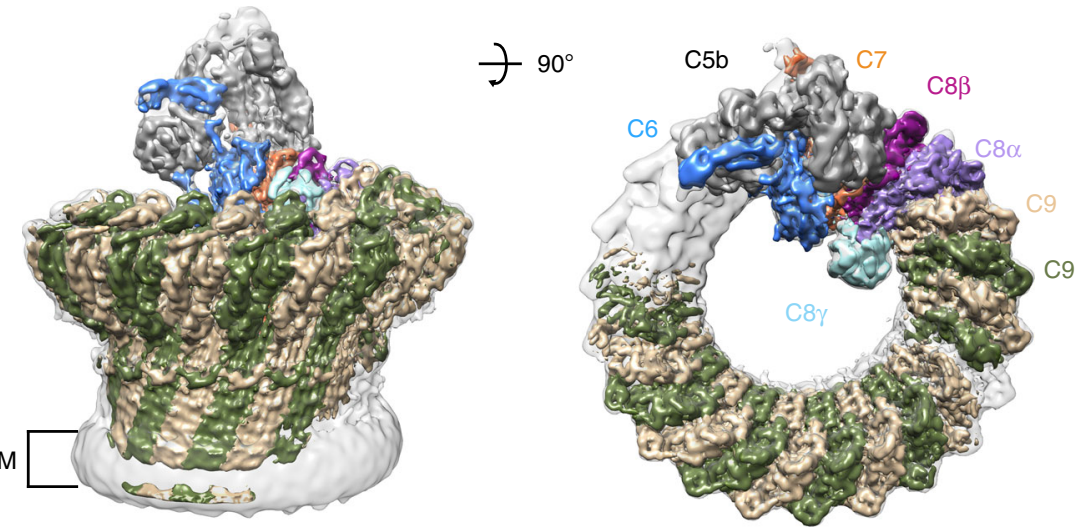

b
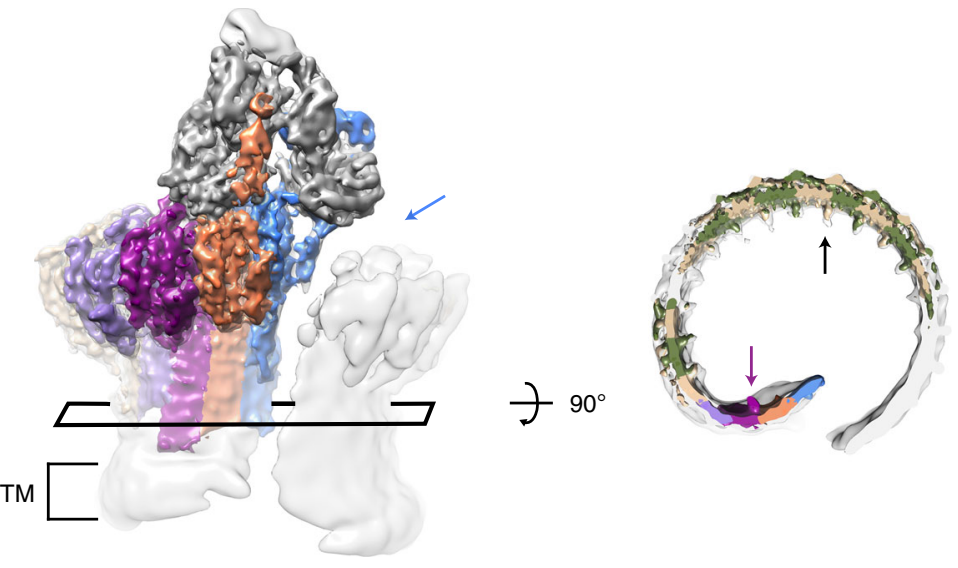

C
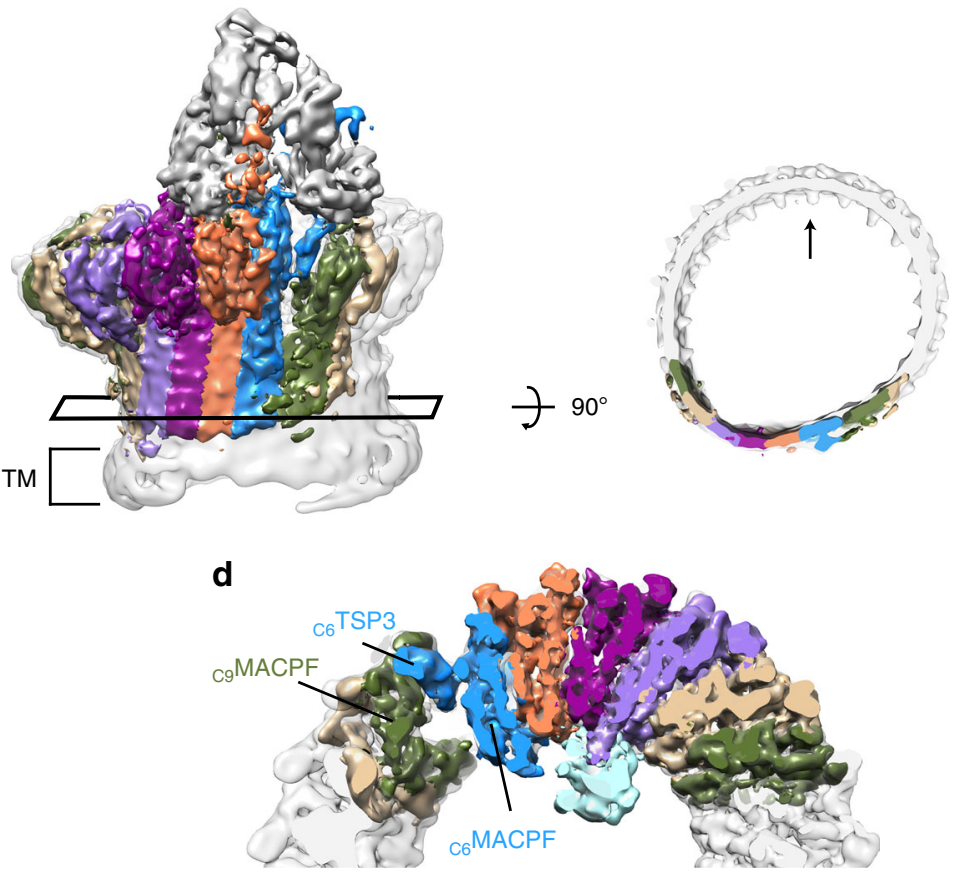

Fig. 1 CryoEM structures of MAC reveal conformational flexibility of the pore. a CryoEM density map for the open conformation (gray transparent surface) overlaid with subvolume reconstructions of the asymmetric, hinge and C9 oligomer regions (colored according to protein components). Transmembrane region is indicated (TM). b CryoEM reconstructions in a rotated $180^{\circ}$. Blue arrow indicates the gap between $\mathrm{C} 6$ and terminal $\mathrm{C} 9$ of the split washer. A rectangle highlights a cross-section of the MAC $\beta$-barrel shown in the right panel. Black arrow highlights density for C9 glycans that protrude into the lumen of the barrel. Purple arrow indicates density for N-linked glycosylation of C8 (N189) within the hinge. c CryoEM map of the closed conformation (gray transparent surface) overlaid with the subvolume reconstruction of the asymmetric region (colored density) in the same orientation as $\mathbf{b}$. Rectangle indicates the cross-section shown in the right panel. d Closed conformation of the MAC slabbed through the core MACPF domains. C6 TSP3 and MACPF domains of $\mathrm{C} 6$ and $\mathrm{C} 9$ are indicated 

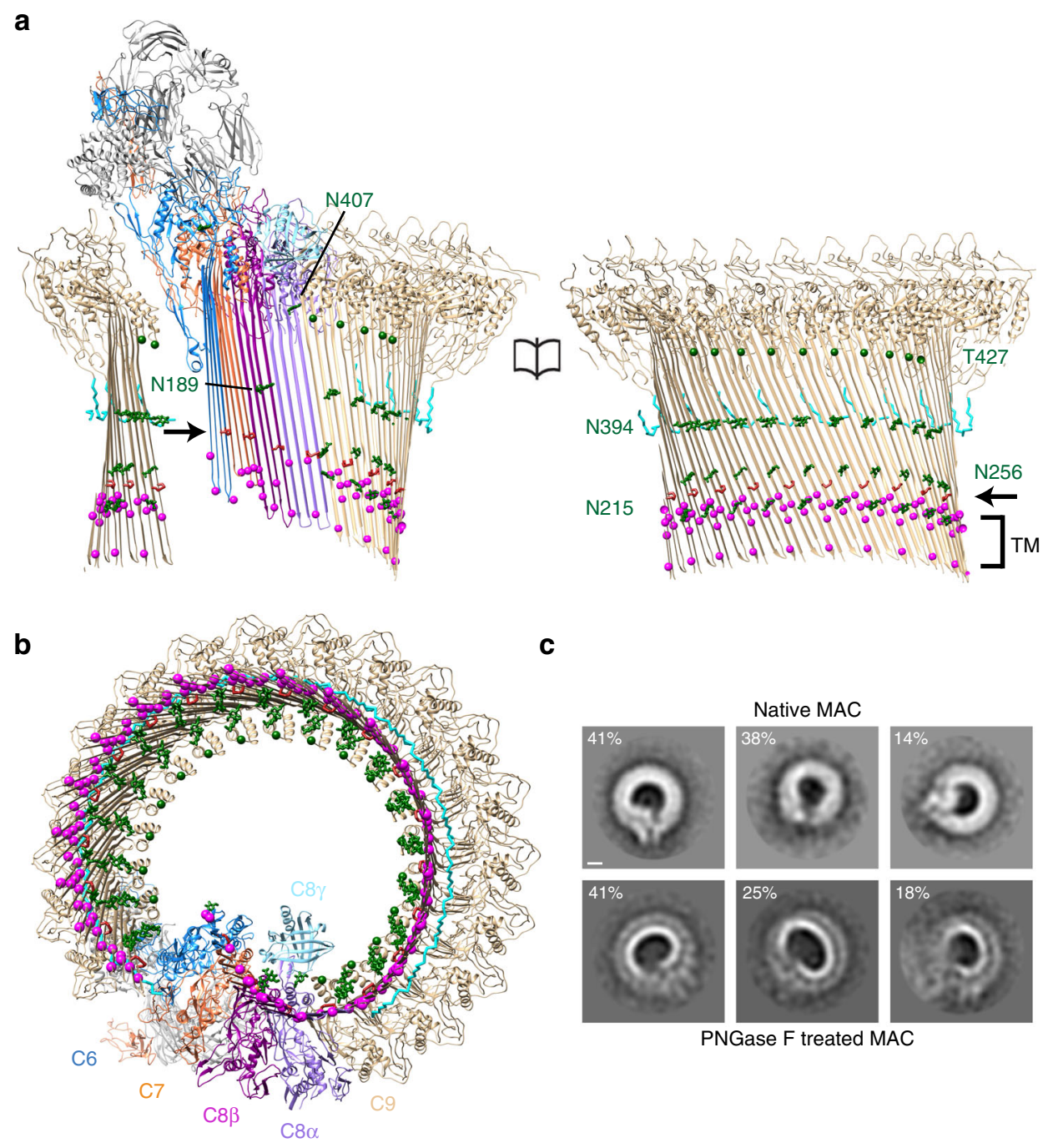

C

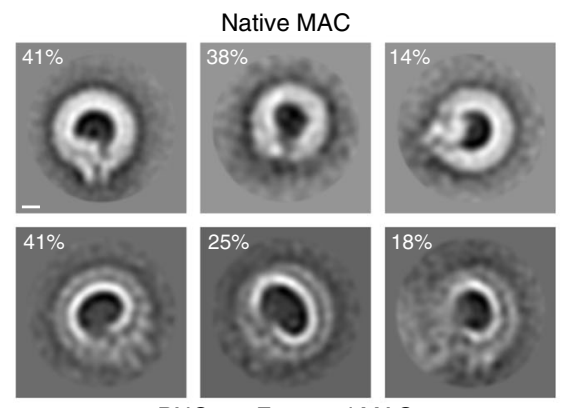

PNGase F treated MAC

Fig. 2 Glycans provide structural support for a giant $\beta$-barrel. Molecular model for the open conformation as viewed from within the $\beta$-barrel (a) and from the membrane (b). N-linked glycans (green) are shown as sticks (C9:N215, N256, N394; C8ß:N189; and C8 $\alpha$ :N407). C $\alpha$ carbons of O-linked C9:T427 are represented as green spheres. Backbone trace of C9 C-terminus (res 522:535) is shown as cyan sticks. Disulfide bonds within MACPF domains are red and indicated by an arrow. $\mathrm{C} \alpha$ carbons of aromatic residues near the membrane (TM) are magenta spheres. c Reference-free 2D class averages of negatively stained MAC assembled from de-glycosylated C8 and C9 (bottom panel) show distorted pores on lipid monolayers as compared to native MAC (top panel). Classes are derived from similar particle numbers to enable a more direct comparison of curvature distortion (native, 706; de-glycosylated, 711). Native MAC particles are a randomly chosen subset of the data shown in Supplementary Fig. 2a which include variations across open and closed conformations. Percentage of particles belonging to each class is indicated. Scale bar, $50 \AA$

C5b6 is a spatial platform for MAC assembly. Limited resolution of previous MAC reconstructions ${ }^{15,16}$ prevented any structural analysis of rearrangements within $\mathrm{C} 5 \mathrm{~b} 6$ during pore formation. We therefore investigated how C7-binding to C5b6 triggers the lipophilic transition using our high-resolution maps (Fig. 3). Our data reveal that the C5b6 complex serves as a spatial platform directing MAC assembly. C5b MG domains 1, 4, and 5, together with the "link" domain, bridge a cluster of arches comprised of the lipoprotein receptor class A (LDL) domains of C6, C7, and C8 $\beta$ (Fig. 3a and Supplementary Fig. 5). While the core of C5b remains largely unchanged during the transition, C6 undergoes marked domain rearrangements upon integration into the MAC.

C6 is comprised of 10 individual domains that can be classified into three functional parts: (1) those that mediate the interaction with $\mathrm{C} 5 \mathrm{~b},(2)$ regulatory auxiliary modules, and (3) the poreforming MACPF domain. Large structural re-arrangements of C6 auxiliary domains accompany conformational changes within the pore-forming MACPF (Fig. 3d and Supplementary Fig. 5a).
Superposition of the soluble and MAC-incorporated forms of $\mathrm{C} 5 \mathrm{~b} 6$ show that although the relative orientation of the $\mathrm{C} 5 \mathrm{~b}$ thioester-like domain (TED) and C6 C-terminal complement control protein (CCP) domains remains unchanged, the C6 LDL is displaced by C7-binding. The two N-terminal TSP domains (TSP1 and TSP2) undergo a concerted rotation with respect to the core MACPF, resulting in a final position near-perpendicular to the plane of the membrane, stabilizing the newly formed $\beta$ sheet. Rotations of regulatory auxiliary modules coincide with an unbending and untwisting of the C6 MACPF $\beta$-sheet. Movement of the C6 epidermal growth factor (EGF) domain and MACPF helix-turn-helix (CH3) motif release the pore-forming $\mathrm{TMH}$ regions, in agreement with lower resolution structures of poreforming toxin homologs 28 .

Auxiliary domains mediate the lipophilic transition. We next explored whether conformational changes incurred by C6 were 
a

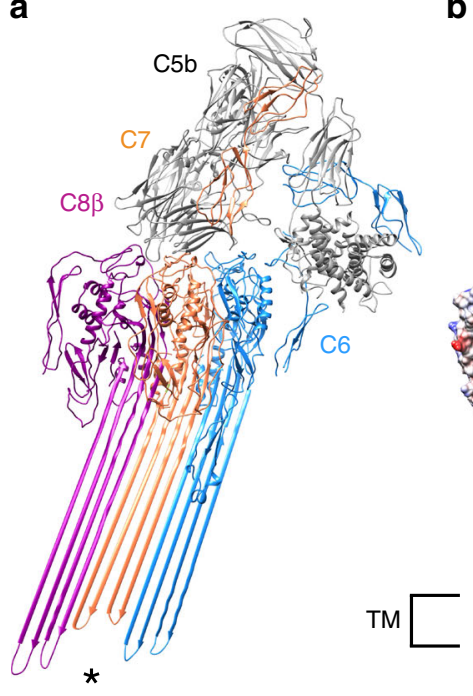

b

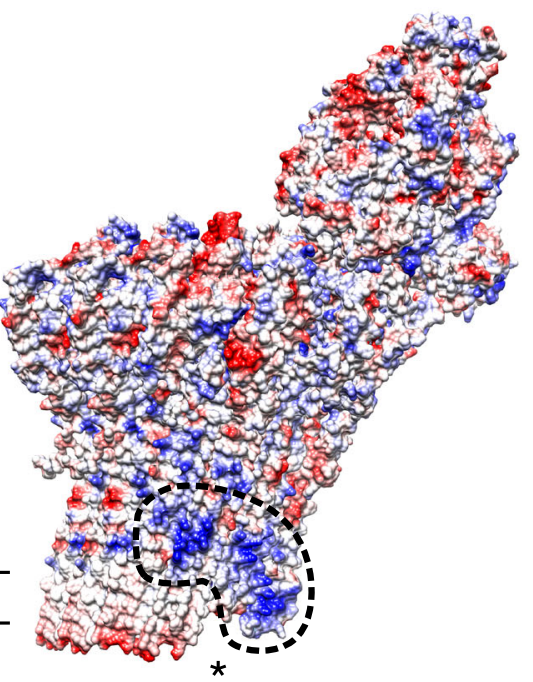

C
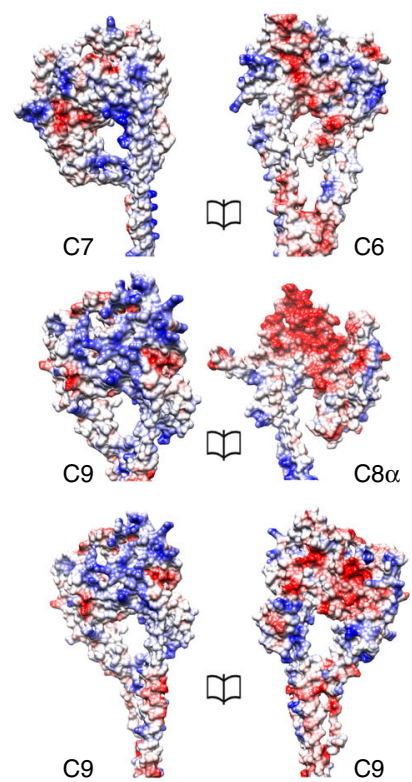

e

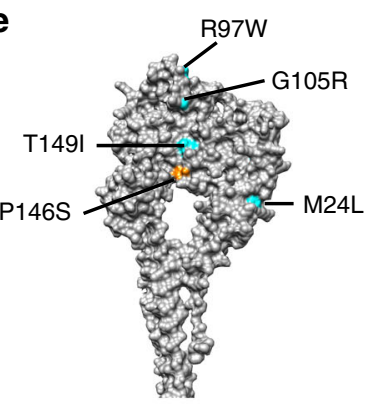

d

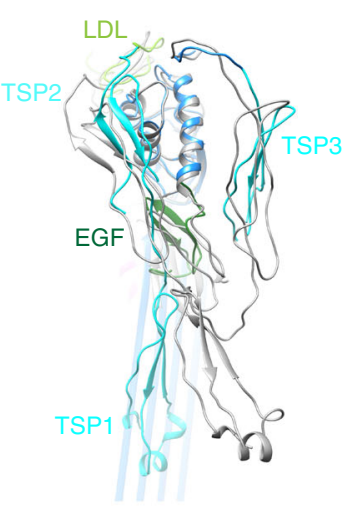

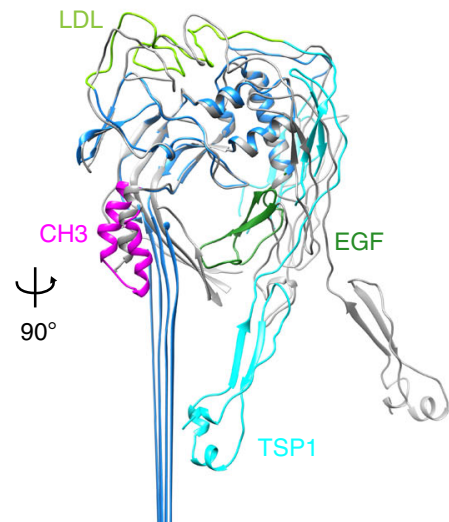

Fig. 3 Structural transitions of complement proteins upon MAC assembly. a Molecular model of complement proteins that interact with C5b (gray). * indicates uneven lengths of TMH regions of C6 (blue), C7 (orange), and C8 $\beta$ (magenta). b Coulombic surface potential (from red $-10 \mathrm{kcal} / \mathrm{mol}{ }^{\star} \mathrm{e}$ to blue $+10 \mathrm{kcal} / \mathrm{mol}^{\star} \mathrm{e}$ ) of atomic models for C5b, C6, C7, C8 and two adjacent C9 monomers viewed from the convex face of the barrel (same view as in a). Dotted line highlights a patch of positively charged residues within the membrane-interacting regions of C6, C7, and C8. Transmembrane regions of C8 and $C 9$ are indicated (TM). * indicates the same region as in a. c Coulombic surface potential representation for MACPF interaction interfaces of complement proteins. d Superposition of the MAC form of C6 (colored) and C6 from the soluble C5b6 crystal structure lacking TMH residues (PDB: 4A5W; gray). Rotations in C6 TSP (cyan), LDL (light green), and EGF (dark green) domains accompany movement of CH3 latch (magenta) and unfurling of transmembrane $\beta$-hairpins. e Genetic variants identified in AMD patients mapped on the C9 structure. Mutations reported to decrease polymerization are cyan, those reported to cause spontaneous self-polymerization are orange $\mathrm{e}^{30}$

conserved across complement proteins. $\mathrm{N}$ - and C-terminal TSP domains of C7, C8 (TSP1 and TSP2), and C9 (TSP1), overlay with those of C6 (TSP2 and TSP3) in its membrane-inserted "active" conformation (Supplementary Fig. 6a). Furthermore, the position of $\mathrm{CH} 3$ relative to the MACPF $\beta$-sheet is the same for all MAC components (Supplementary Fig. 6b). Although the core MACPF domains of all MAC components overlay well, the glycine-rich hinge enables a wide range of angles to accommodate the varied curvature of the barrel. Similar to the pore transition of C6, C8 regulatory auxiliary modules (EGF and TSP2) release TMH regions of both $\alpha$ and $\beta$ chains (Supplementary Fig. 6c). However, unlike the dramatic C6 MACPF unbending, the angle of the C $8 \beta$ MACPF sheet remains constant (Supplementary Fig. 6d). Component strands untwist to align with the $\mathrm{C} 7 \beta$-sheet and pack against the $\mathrm{C} 7 \mathrm{CH} 3$ latch. Surprisingly, $\mathrm{C} 8 \beta \mathrm{CH} 3$ does not undergo a lateral shift during pore formation, suggesting that the C8 $\beta$ MACPF is already primed for membrane insertion.
To investigate the molecular basis underpinning the directionality of MAC's sequential assembly, we compared electrostatic surface potentials of complement proteins (Fig. 3c). The interface between $\mathrm{C} 6-\mathrm{C} 7, \mathrm{C} 8 \mathrm{a}-\mathrm{C} 9$, and $\mathrm{C} 9-\mathrm{C} 9$ is formed mainly by polar and charge interactions (Supplementary Table 2). Incorporation of $\mathrm{C} 8 \alpha$ exposes a negatively charged patch that may influence recruitment of the positively charged face of C9. C9 oligomerization could be propagated by electrostatic complementarity between leading and lagging faces, consistent with CDC pore formation $^{29}$. Mutations that alter polymerization of C9 are implicated in some cases of age-related macular degeneration $(\mathrm{AMD})^{30}$. We are now able to assign disease-related variants on the human C9 structure, and find that three of these AMDassociated mutations (P146S, G105R, and T149I) would likely impact the negatively charged patch that drives oligomerization. G105R and T149I variants decrease polymerization of $C 9^{30}$ and would reduce the footprint of the negatively charged interface. By 
contrast, variant P146S increases self-polymerization of $\mathrm{C} 9^{30}$ and may act by increasing polarity of the surface.

Interactions with the lipid bilayer. Complement proteins within the MAC interact with the lipid bilayer in two distinct ways. Transmembrane hairpins of MAC components vary in length and amino acid composition. $\mathrm{C} 6$ and $\mathrm{C} 7$ hairpins contain a single band of aromatic residues (Fig. 2a). A patch of positively charged residues resides proximal to the tips of the short C6 and C7 $\beta$ hairpins (Fig. 3a, b), suggesting interaction with phospholipid headgroups of the outer leaflet. Similar to other $\beta$-barrel pore assemblies $^{31}$, two rings of aromatic residues separated by the width of the bilayer anchor C8 and C9 within the membrane (Fig. 2a). The longer hairpins of C8 and C9 expose hydrophobic residues on the $\beta$-barrel's outer surface (Fig. 3b), consistent with an interface with lipid tails.

To investigate how these two types of interactions impact mechanical properties of the target cell membrane, we performed fluctuation analysis (flicker spectroscopy) of giant unilamellar vesicles (GUVs). In flicker spectroscopy, the magnitude of thermally induced fluctuations in a GUV membrane are quantified by tracking the membrane contour in a series of video-microscopy images. This data is fitted to a two-parameter model to extract the membrane bending rigidity and tension ${ }^{32}$. We used phase contrast light microscopy to track individual GUVs at each step of MAC assembly and confirmed the presence of functional pores by diffusion of sucrose across the bilayer (Fig. 4a). We recorded membrane fluctuations after the addition of C5b6, C7, C8, and C9, or when buffer replaced C5b6 in the sequence. When C5b6 was absent, the amplitude of the GUV membrane fluctuations remained constant throughout the experiment (Fig. 4a, b and Supplementary Movie 2). The small increase in fluctuations observed at later time points is likely to be due to slight increases in temperature caused by lamp heating. By contrast, membrane fluctuations of GUVs that supported MAC assembly were reduced upon C8-binding and were diminished to negligible levels with the addition of C9 (Fig. 4a, b and Supplementary Movie 2). These data suggest that large MAC lesions in cells may lead to rigidification of the membrane by the insertion of $\beta$-hairpins across the bilayer and by equilibration of the extracellular milieu across the pore.

Where fluctuation amplitudes could be parameterized, we analyzed changes in bending modulus and tension during MAC assembly (Fig. 4c and Supplementary Fig. 7). Bending modulus is an intrinsic property of the membrane and describes the amount of energy required to change the mean curvature of a lipid bilayer. It can be affected by thickness of the bilayer ${ }^{33}$, membrane asymmetry $^{34}$, and lipid composition ${ }^{35}$. C5b6 is a soluble complex that ionically associates with membranes ${ }^{36}$. Upon C7-binding, the complex becomes lipophilic and is irreversibly tethered ${ }^{12}$. Although this study and previous work are consistent with an interaction of $\mathrm{C} 7$ with lipids ${ }^{12,37,38}$, the ability of C5b6 to alter the biophysical properties of the membrane was unanticipated. C5b6 reduced the bending modulus of GUVs without affecting tension (Fig. 4c and Supplementary Fig. 7). The reduction in bending modulus was maintained upon $\mathrm{C} 7$ binding and anchoring of the C5b7 complex to the GUV. As C8 incorporates into the MAC to form C5b8, bending modulus increases (Fig. 4c). By comparison, tension remains largely unchanged (Supplementary Fig. 7a). Taken together, MAC specifically impacts the energy required to bend the membrane in a way that changes during the sequential assembly pathway. While our experimental system assumes a uniform distribution of pores on GUVs, MAC formation on target cells is heavily influenced by activation of upstream complement pathways and opsonization of bacterial cells. These deposition hotspots may therefore influence changes in bending rigidity coefficients in a concentration-dependent manner.

\section{Discussion}

We have presented here cryoEM structures of two MAC conformations and derived an atomic model for the transmembrane complex. Furthermore, we have shown corroborating flicker spectroscopy data that explain how MAC assembly precursors alter physical properties of the membrane to prime the bilayer for attack. Our results provide a structural framework on which to interpret earlier biological data and inform future mechanistic models. Our structure has revealed a number of features that increase our understanding of the complement system, and informs a general mechanism for how $\beta$-barrel pore-forming proteins rupture lipid bilayers.

Previous high resolution structures of $\beta$-pore forming proteins were based on the assumption that the oligomeric assemblies are both rigid and symmetric ${ }^{29,39}$, however we discovered that these proteins can be flexible within the membrane. Here we demonstrate that complement proteins within the C5b8 paddle rotate with respect to the nascent $\mathrm{C} 9$ oligomer, which in itself varies in curvature between the two observed MAC conformations (Supplementary Movie 1). A glycan scaffold that lines the inner wall of the giant $\beta$-barrel constrains the range of movement (Fig. 2). Together with $\mathrm{C} 8 \gamma$, wedged between $\mathrm{C} 5 \mathrm{~b} 8$ and $\mathrm{C} 9$, this scaffold provides a structural support that maintains the curvature of an otherwise unsupported tall and flexible giant $\beta$-barrel pore. We propose that rotation of pore $\beta$-hairpins within the bilayer could provide an additional level of membrane destabilization that contributes to lytic activity of $\beta$-pore forming proteins. Bacterial membranes are complex targets, densely packed with both polysaccharides and porins ${ }^{40}$. Flexibility of a growing MAC pore would allow short stretches of $\beta$-hairpins to move within the plane of the bilayer to accommodate the compositional complexity of its membrane environment.

One of the most striking observations from our structure is that the MAC pore is comprised of $\beta$-hairpins that differ in both length and charge properties. These differences provide the structural basis for how complement proteins interact with target cells. Partially inserted $\beta$-hairpins of C6 and C7 interact with lipid headgroups of the outer leaflet and decrease the bending modulus of the membrane (Fig. 4). Subsequently, the increase in membrane stiffness we observe upon $\mathrm{C} 8$ addition could be attributed in part to the mechanical strain of distorting the bilayer. Membrane interacting $\beta$-hairpins of $\mathrm{C} 6, \mathrm{C} 7$, and neighboring $\mathrm{C} 8 \beta$ differ in length (Fig. 3a) and likely bend the bilayer to form the non-lamellar edge of an arc-pore. Levels of MAC on the plasma membranes of self-cells are restricted by endocytic pathways mediated by changes in the mechanical properties of the lipid bilayer ${ }^{41,42}$. This mechanism plays an important role in cell activation pathways ${ }^{41}$, but also in the immune response to cancer ${ }^{9}$. In our model, we propose that differences in charge and length of the pore $\beta$-hairpins impact physical properties of the lipid environment that could stimulate activity of mechanosensors, such as caveolin-1 to facilitate MAC removal and recovery from MAC attack. Similarly, membrane repair mechanisms triggered in response to bacterial pore-forming toxins may rely on changes in mechanochemical properties of the plasma membrane caused by oligomerized prepores ${ }^{43}$. Although pore forming hairpins of these bacterial homologs extend throughout the bilayer, we speculate that an auxiliary domain's cholesterol recognition loops that interact with the outer leaflet may serve a similar role as the partially-inserted MAC precursor.

Whereas this study focuses on how MAC interacts with the membrane, C8 and C9 also bind CD59 to block complement- 
a
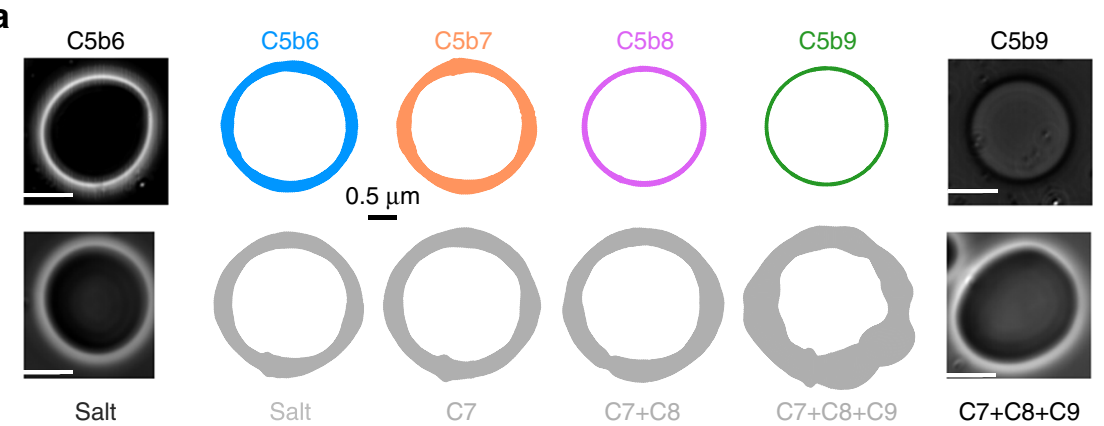

Salt

Salt

C7

$\mathrm{C} 7+\mathrm{C} 8$

$\mathrm{C} 7+\mathrm{C} 8+\mathrm{C} 9$

$\mathrm{C} 7+\mathrm{C} 8+\mathrm{C} 9$

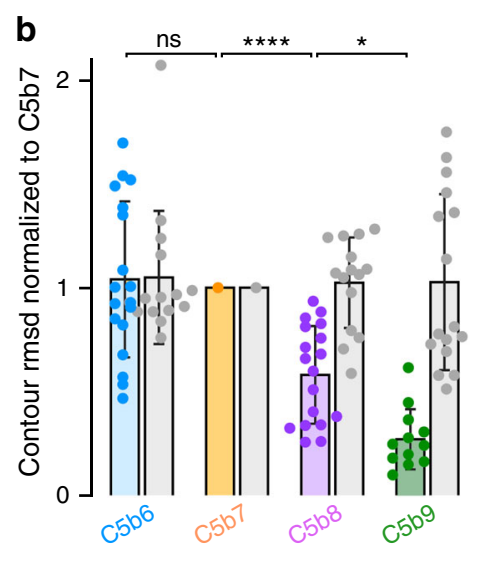

c

d

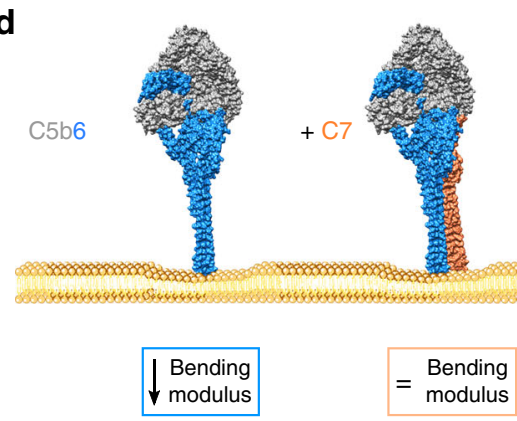

Easier to bend membrane
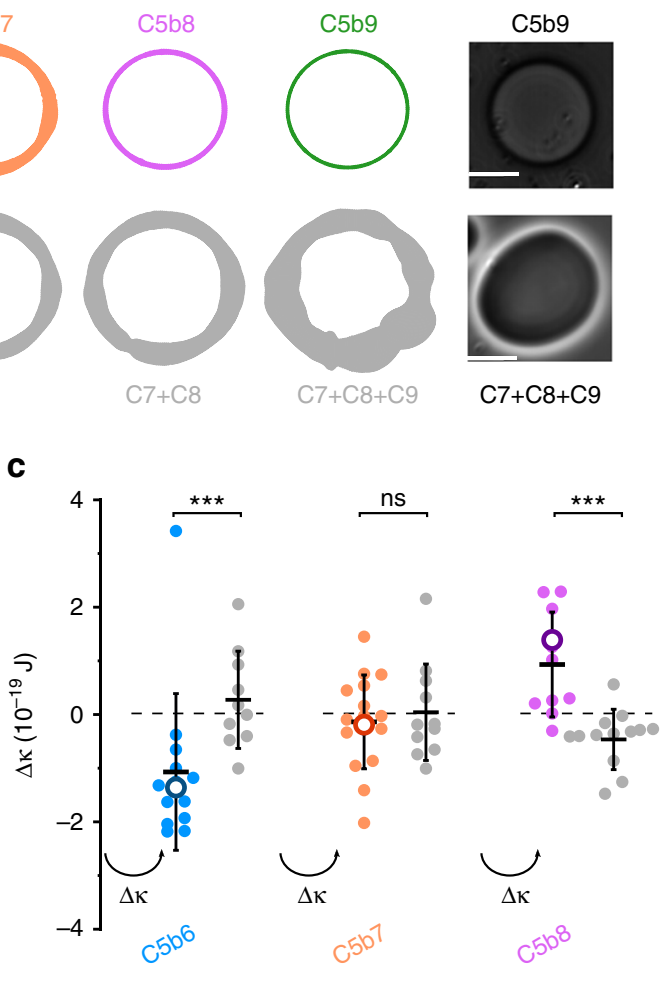

\section{(1)}


Our structural and biophysical data inform a general mechanism for $\beta$-pore forming proteins (Fig. 4d). Large-scale rotations of regulatory domains accompany the aqueous-totransmembrane transition of soluble monomers. We propose that partially inserted proteins initiate interaction with the outer leaflet and prime the membrane for attack by lowering the energy for bending the lipid bilayer, a mechanism exploited by malarial parasites during cell invasion ${ }^{49}$ and by the insertion and folding of $\beta$-barrel proteins ${ }^{50}$. Subsequent protein conformational rearrangements distort the lamellar structure of the bilayer, causing it to stiffen and ultimately rupture. Changes in mechanical properties of the lipid environment could explain the nuanced cellular response to membrane damage by both MAC and bacterial pore-forming toxins.

In conclusion, we show that the MAC is a flexible immune pore that interacts with the lipid bilayer in two distinct ways. These interactions govern how MAC initiates membrane-binding and ultimate rupture. Our observations provide a general mechanism for pore formation and explain how MAC function can be tuned to different targets and cellular contexts.

\section{Methods}

Purification of MAC pores. A lipid mix consisting of 1,2-dioleoyl-sn-glycero-3phosphocholine (DOPC) and 1,2-dioleoyl-sn-glycero-3-phosphoethanolamine (DOPE) (6:4 w/w, Anatrace) in chloroform was dried under nitrogen gas and rehydrated in buffer containing $20 \mathrm{mM}$ HEPES-NaOH, $150 \mathrm{mM} \mathrm{NaCl}$ at $\mathrm{pH}$ 7.4. Rehydrated liposomes were extruded through a $100 \mathrm{~nm}$ polycarbonate membrane (Whatman) to produce a monodisperse solution of unilamellar liposomes, whose local membrane curvature is negligible on the length scale of the complete pore. Complement proteins C5b6, C7, C8, and C9 (Complement Technology) were sequentially incubated with liposomes at a molar ratio of 1:1:1:18. A 5 min incubation step was allowed between each component addition followed by incubation for $1 \mathrm{~h}$ at $37^{\circ} \mathrm{C}$ to optimize for assembly completion before transferring to $4{ }^{\circ} \mathrm{C}$ overnight. Assembled MAC complexes were solubilized in $1.5 \%$ Cymal-5 (Anatrace) in the presence of DOPC $(1 \mathrm{mg} / \mathrm{ml})$ and glycerol $(10 \%)$ for 1 hour at room temperature. Solubilized complexes were purified using density centrifugation in a sucrose solution (5-20\%) containing 0.004 \% Cymal-7 NG (Anatrace). Samples were spun for 4 hours at 45,000 rpm using an SW60Ti rotor. Fractions were screened using negative stain EM and those containing complete MAC pores were pooled, concentrated and sucrose removed using a ZebaSpin desalting column (Thermofisher Scientific).

De-glycosylation of C8 and C9. C8 and C9 were incubated with PNGase F (NGlycosidase F, Roche) at the ratio $1 \mu \mathrm{g}: 0.67 \mathrm{U}$ and diluted in protein buffer $(120 \mathrm{mM} \mathrm{NaCl}, 20 \mathrm{mM}$ Hepes $\mathrm{pH} 7.4)$ to a final concentration of $0.3 \mathrm{mg} / \mathrm{ml}$. Under control conditions C8 and C9 were mixed in identical ratios with buffer (50 $\mathrm{mM}$ sodium phosphate $\mathrm{pH} 7.3,12.5 \mathrm{mM}$ EDTA, 50\% glycerol) and diluted to $0.3 \mathrm{mg} / \mathrm{ml}$. Reaction mixes were incubated for $48 \mathrm{~h}$ at $37^{\circ} \mathrm{C}$.

Liposome lysis assay. A DOPC:DOPE lipid mix (6:4 mol:mol) was resuspended in calcein solution ( $50 \mathrm{mM}$ calcein, $150 \mathrm{mM} \mathrm{NaCl}, 20 \mathrm{mM}$ Hepes pH 7.4), freezethawed 6 times $\left(-196^{\circ} \mathrm{C}\right.$ to $\left.65^{\circ} \mathrm{C}\right)$ and extruded through a $100 \mathrm{~nm}$ polycarbonate membrane to form calcein encapsulated liposomes. Liposomes were purified on a gravity flow Sephadex-G50 (GE Healthcare) column to remove non-encapsulated calcein. Calcein is self-quenched at $50 \mathrm{mM}$ and is un-quenched through its dilution in the outer solution following MAC lysis of liposomes. Liposomes lysis was performed by sequential addition of C5b6 $\left(5 \mathrm{~min}, 37^{\circ} \mathrm{C}\right), \mathrm{C} 7\left(5 \mathrm{~min}, 37^{\circ} \mathrm{C}\right), \mathrm{C} 8$ and $\mathrm{C} 9$ at a mass ratio of 1:1:1:1. Fluorescence was recorded immediately following C9 addition and every minute for $60 \mathrm{~min}$ on a SpectraMax M2 fluorometer (Molecular Devices) with monochromator excitation at $490 \mathrm{~nm}$ and emission recorded at 520 $\mathrm{nm}$. For each independent recording, background fluorescence (calcein encapsulated liposomes alone) was measured and subtracted from the data before normalizing to the maximal fluorescence value. Maximal lysis fluorescence was recorded after bursting the liposomes through a freeze-thaw cycle. MAC lysis of liposomes was measured in three independent replicates.

Negative stain EM of MAC pores on lipid monolayers. To form lipid monolayers, $4 \mathrm{~mm}$ diameter wells in a Teflon plate were filled with $9 \mu \mathrm{l}$ of buffer $(120 \mathrm{mM} \mathrm{NaCl}, 20 \mathrm{mM}$ Hepes pH 7.4) and overlaid with $2 \mu \mathrm{l}$ of DOPC:DOPE (60:40 mol:mol) at $1 \mathrm{mg} / \mathrm{ml}$ in chloroform. Chloroform was allowed to evaporate for $1 \mathrm{~min}$ and a CF400-CU grid (Agar Scientific) was deposited at the surface of the well with the carbon side facing the solution. To form MAC pores, all steps were performed at $37^{\circ} \mathrm{C}$ and the Teflon plate was kept on buffer soaked tissue in a closed container to maintain constant humidity. With minimal perturbation to the monolayer C5b6, C7, C8 (60 nM final concentration), and C9 (1.2 $\mu \mathrm{M})$ were sequentially added to the solution at molar ratios of 1:1:1:20. A 5 min incubation step was allowed between each component addition followed by $15 \mathrm{~min}$ incubation after C9 addition. Grids with adherent monolayers were then gently peeled off the solution and directly stained in uranyl acetate $2 \%$ (w:v). Images were acquired on a Tecnai F20 electron microscope (Thermo Fisher Scientific) with a Falcon II camera at $\times 50,000$ magnification $(2.05 \AA$ pixel), $0.75-1.5 \mu \mathrm{m}$ underfocus. Grids were imaged across 4 quadrants of the grid to control for local variations in monolayer composition. For PNGase F digested samples, images were acquired on a Tecnai T12 electron microscope (Thermo Fisher Scientific) with a F216 camera (TVIPS) at $\times 42,000$ magnification (3.71 ̊̊/pixel).

CryoEM. In order to obtain a sufficient proportion of intact pores on cryoEM grids, freshly purified solublized MAC $(2.5 \mu \mathrm{l})$ was applied to glow-discharged holey carbon grids (Quantifoil R 1.2/1.3). Samples were flash frozen in liquid ethane cooled in liquid nitrogen using a Vitrobot Mark III (Thermo Fisher Scientific) and stored under liquid nitrogen until use. Screening of cryoEM conditions was performed on a $120 \mathrm{kV}$ Tecnai T12 (Thermo Fisher Scientific). Eight datasets were collected on $300 \mathrm{kV}$ Titan Krios microscopes (Thermo Fisher Scientific) equipped with Falcon II or Falcon III direct electron detectors (Thermo Fisher Scientific), at a defocus range of 1.75 to $4 \mu \mathrm{m}$ underfocus. Exposures were recorded as movies comprising of 32-39 frames. Due to the flexibility and low concentration of detergent solubilized pores, large datasets were collected to obtain sufficient populations of homogenous particles. Particle distribution was highly dependent on ice-thickness, therefore only holes that allowed us to obtain both sufficient contrast and monodisperse particles were selected. Some datasets included the use of carbon-coated holey carbon grids to improve the distribution of particles. A summary of imaging conditions is provided in Supplementary Table 1.

Image processing. Electron micrograph movie frames were aligned by MotionCor $2^{51}$, discarding the first and last frames. CTF parameters were estimated using CTFFIND4 ${ }^{52}$. Any movies containing low figure of merit scores, substantial drift, low contrast, thick ice, or crystalline ice were discarded from further analysis. Particles were manually selected and extracted from high-quality aligned movies using RELION ${ }^{53}$. Particles were subjected to iterative rounds of $2 \mathrm{D}$ classification to improve the homogeneity of the dataset. The published MAC reconstruction was strongly low-pass filtered ( $60 \AA$ ) to prevent model bias and used as a starting model for a gold-standard 3D autorefinement of images. 231,767 selected particles contributed to a consensus MAC structure whose resolution was determined at $4.9 \AA$ These orientations served as the starting point for tracking beam-induced movement of individual particles, which was corrected using particle polishing within RELION.

3D classification of images revealed conformational heterogeneity of the MAC (Supplementary Fig. 1). Particles containing an intact closed $\beta$-barrel (23\%) were grouped and subjected to an additional round of 3D autorefinement (5.6 resolution). Those that were stoichiometrically identical to the closed conformation but had an open $\beta$-barrel (35\%) were grouped separately and independently refined (5.6 ̊ resolution). It was not possible to improve the maps as a whole for the two conformations because of a continuous relative rotation between the asymmetric region (C5b6, C7, C8, and neighboring C9) and the $\mathrm{C} 9$ oligomer. We therefore solved the structures of the asymmetric region $(4.7 \AA$ and $5.9 \AA$, open and closed respectively), hinge of the open conformation (4.9 $\AA$ ), and C9 oligomer of the open conformation $(4.4 \AA)$ separately. The global open and closed maps were used as a reference to assemble the parts together. To improve the alignment of the asymmetric and hinge regions, density corresponding to the C9 oligomer was subtracted from the original images. Orientation parameters for the asymmetric and hinge regions were refined from density-subtracted images by applying a mask based on its position in maps generated from the original images. Masks were optimized to include regions of the structure that moved together and were included in subsequent rounds of 3D autorefinement. A similar procedure was adopted to focus the refinement on the $\mathrm{C} 9$ oligomer. A final resolution of $4.4 \AA$ was achieved for the $\mathrm{C} 9$ oligomer within the open conformation by averaging density for 8 neighboring copies in Chimera ${ }^{54}$. Resolution of all maps was determined using the masking-effect corrected Fourier Shell Correlation (FSC) as implemented in RELION post-processing. Local resolution estimates were calculated within a soft spherical mask that is translated across the map, using phase-randomization to assess the convolution effects of the mask and locally low-pass filtered, as implemented within RELION.

Model building and refinement. Crystal structures for soluble $\mathrm{C} 5 \mathrm{~b} 6^{11}$, and $\mathrm{C} 8^{21}$ together with homology models for C7 and C9 were used as initial models for refinement into local resolution filtered maps. Due to differences in map quality between the two conformations, the asymmetric and hinge regions of the open state were used for refinement of C5b6, C7, and C8. C9 was refined into the locally averaged $\mathrm{C} 9$ oligomer of the open conformation, and placed as a single rigid body in the remaining 17 copies within the map. TMH regions of $\mathrm{C} 6$ and $\mathrm{C} 8$ were removed from the soluble structures prior to fitting, as these are known to undergo a structural transition to form $\beta$-hairpins. Domains of C5b (C345C and MG8), C6 (FIM1 and FIM2), and C7 (FIM1 and FIM2) for which there was weak or no 
density were also removed from the model. Truncated crystal structures for C5b6 and $\mathrm{C} 8 \alpha \beta \gamma$ were initially placed manually in to the map using Chimera followed by rigid body fitting using the Fit in Map tool. These atomic models were split into domains, which were real-space refined as rigid bodies in $\operatorname{Coot}^{55}$. Domains that comprised the primary interaction interface between $\mathrm{C} 5 \mathrm{~b}$ and $\mathrm{C} 6$ were grouped as a single body (C6: CCP1, CCP2, TSP3, and C5b: TED) in the first instance. C5b MG domains were also grouped in the early stages of refinement. As there are no crystal structures available for $\mathrm{C} 7$ or $\mathrm{C} 9$, homology models were generated using MODELLER $^{56}$. C8 $\beta$ was chosen as template for C9 (26.76\% sequence identity), while the C7 model was based on the crystal structure of soluble C6 $31.87 \%$ sequence identity) together with coordinates for the $\mathrm{C} 7$ Factor I-like domains (PDB:2WCY $)^{57}$. $\mathrm{CH} 3$ helices of MAC proteins were remodeled with MODELLER and the $15 \mathrm{C}$-terminal residues of $\mathrm{C} 9$ were manually built in Coot. For C9, the LDL, EGF, and TSP domains were flexibly fitted in a stepwise, iterative process whereby large-scale movements were refined first using iMODFIT ${ }^{58}$ followed by further local real-space refinement using Flex-EM ${ }^{59}$. Here, segments of the structure were restrained based either on user-defined rigid bodies or those defined by RIBFIND $^{60}$. The fitting progress was analyzed by local scoring using the Segment Based Manders' Overlap Coefficient (SMOC) ${ }^{61}$, as implemented in the TEMPy software ${ }^{62}$. Refinement was carried out iteratively until the CCC between the map and model stabilized. Once each component was fitted, interfaces were assessed for clashes with Chimera followed by further refinement of the sub-complexes, as necessary. Loops connecting domains were refined in Coot or removed if density was not apparent. Models were further optimized using PHENIX real-space refinement ${ }^{63}$ with secondary structure element and disulfide bond restraints. Where crystal structures were available, reference-based restraints were also imposed. The MAC pore $\beta$-hairpins were extended with idealized $\beta$-strands, in which main-chain geometry was generated using an in-house program to set amino acid (phi, psi) angles to $(-140,135)$. A full atomic model was then generated by adding side-chains to backbone atoms using the SCWRL4 program ${ }^{64}$. Modeled strands were least squares-fitted onto existing $\beta$-strands of core MACPF domains and manually adjusted to minimize clashes using Coot. Overlapping residues were removed. Models were merged and linking residues were real-space refined in Coot. Known glycosylation sites were added to the extended model in Coot, with placement guided both by residue position and visible density. The final models were subjected to a final refinement using global minimization in PHENIX with secondary structure and di-sulfide bond restraints. The quality of the final fits between maps and models per residue was assessed using TEMPy SMOC score (Supplementary Fig. 3). Statistics of overall model quality and geometry outliers for final models were reported using MolProbity ${ }^{65}$ (Supplementary Table 1).

Map visualization and analysis. Density maps and models were visualized in Chimera. Local resolution of the maps and angular distribution of the particles were assessed in RELION and visualized in Chimera. Coulombic potentials of interaction interfaces were calculated and visualized in Chimera. Maps sharpened with a global B-factor and low pass filtered according to local resolution estimates were used for fitting and refinement. Interaction interfaces and structural rearrangements of complement proteins were analyzed in Coot. Structural movie and figures were generated in Chimera.

Flicker spectroscopy. DOPC:DOPE (60:40 mol:mol) was dissolved at $1 \mathrm{mg} / \mathrm{ml}$ in chloroform and coated onto the conductive side of an indium tin oxide coated glass slide (Sigma-Aldrich). Following chloroform evaporation for $30 \mathrm{~min}$, two glass slides (one coated with lipid, one without) were placed either side of a custommade Polydimethylsiloxane (PDMS) o-ring (Sigma-Aldrich) with the conductive sides of the slides facing inwards to form a chamber. The chamber was filled with a solution of $290 \mathrm{mM}$ sucrose, $1 \mathrm{mM}$ Hepes $\mathrm{pH} 7.4$ and the conductive slides were connected to a TG315 signal generator (Aim-TTi Instruments). To electro-form GUVs, an alternating potential of $1 \mathrm{~V}$ at $10 \mathrm{~Hz}$ was applied through the slides for 2 $\mathrm{h}$, followed by $1 \mathrm{~V}$ at $2 \mathrm{~Hz}$ for $1 \mathrm{~h}$ to detach the GUVs from the glass. The sucrose containing GUVs were then diluted in a hyperosmotic solution of $1 \%$ bovine serum albumin (BSA), $360 \mathrm{mM}$ glucose, $50 \mathrm{mM} \mathrm{NaCl}$, and $10 \mathrm{mM}$ Hepes pH 7.4 and imaged on the day they were produced.

GUVs were imaged in CoverWell perfusion chambers (Grace Bio-Labs) attached to BSA-coated glass slides, allowing the sequential injection of MAC components while continuously tracking individual GUVs. Fluctuation videos were recorded on an Eclipse TE2000-E microscope (Nikon Instruments) at $\times 30$ or $\times 60$ magnification using a Zyla sCMOS camera (Andor) at a frame rate of $\sim 30$ frames per second and an exposure of $0.5 \mathrm{~ms}$. MAC formation was achieved through sequential addition of C5b6, C7, C8, and C9 in 1:1:1:21 molar ratios allowing for a 5 min incubation between each addition. Thirtysecond fluctuation videos were recorded prior to $\mathrm{C} 5 \mathrm{~b} 6$ addition and following the addition and incubation of each component. Loss of GUV contrast was used as an indicator of full MAC deposition and lysis of the GUV, and was observed 8-12 min post C9 addition. GUVs were chosen at random from those that were visibly fluctuating and all GUVs that lysed post-C9 addition were used for analysis ( $>95 \%$ of GUVs). In control experiments, C5b6 was replaced by protein buffer $(120 \mathrm{mM} \mathrm{NaCl}, 10 \mathrm{mM}$ Hepes $\mathrm{pH} 7.4)$; the rest of the protocol was identical.

Analysis of bending modulus and tension variations was performed using a custom-built LabView program (National Instruments) ${ }^{66}$. Briefly, GUV contour coordinates were extracted with subpixel resolution from each video frame and Fourier transformed to extract fluctuation modes. The fluctuation mode amplitudes were averaged across all frames in a particular video to give mean square amplitudes at the GUV equator $\left(h^{2}\left(q_{x} y=0\right)\right)$. These were plotted as a function of the mode wavenumber $\left(q_{x}\right)$ and the following model was fitted to the data to extract bending modulus $(\kappa)$ and tension $(\sigma)$ values as described in equation (1):

$$
h\left(q_{x}, y=0\right)^{2}=\frac{1}{L} \frac{k_{\mathrm{B}} T}{2 \sigma}\left(\frac{1}{q_{x}}-\frac{1}{\sqrt{\frac{\sigma}{k_{c}}+q_{x}}}\right)
$$

where $k_{\mathrm{B}}$ is the Boltzmann constant, $T$ temperature, and $L$ the mean GUV contour circumference. Fluctuation data were fitted from mode 4 to mode 20

(Supplementary Fig. 7b). Failure of fits were mostly a result of contrast loss due to GUV lysis after C9 addition, bending modulus variations were hence never extracted for this step. In some cases, fitting was not possible at earlier steps of MAC formation as a result of poor contour extraction, due to image interference caused by neighboring GUVs or significant deformation of the GUV leading to unreliable contour tracking. For these GUVs, bending and tension were not extracted at the step of fit failure but were analyzed at previous steps and integrated into the data set. In addition, changes in the extent of fluctuation in each GUV were quantified following the addition of each MAC component by calculating the contour RMSD (averaged over all frames for each video).

For all flicker spectroscopy experiments, 18 individual GUVs were tracked through MAC formation over three independent experiments. By measuring changes in bending modulus across the same vesicle throughout the assembly process, these measurements are independent of intrinsic small variations in GUV mechanical properties within a population of vesicles. Control experiments were performed on the same day and same batch of GUVs. As some data sets did not follow a normal distribution, as defined by the D'Agostino \& Pearson and Shapiro-Wilk tests, all variation significances were assessed with double-sided nonparametric tests (Mann-Whitney and Wilcoxon match-pair tests). All statistics were computed using Prism (GraphPad Software), figure plots were generated using DataGraph (Visual Data Tools).

Code availability. Custom software for membrane fluctuation analysis will be provided upon request.

\section{Data availability}

CryoEM data and corresponding atomic models have been deposited in public repositories. Seven maps have been deposited in the Electron Microscopy Data Bank with accession codes: EMD-0106, EMD-0107, EMD-0109, EMD-0110, EMD0111, EMD-0112, EMD-0113. Atomic coordinates have been deposited in the Protein Data Bank with accession codes PDB: 6H03, 6H04. Other data are available from the corresponding author upon reasonable request. A reporting summary for this Article is available as a Supplementary Information file.

Received: 17 August 2018 Accepted: 12 November 2018 Published online: 14 December 2018

\section{References}

1. Morgan, B. P., Boyd, C. \& Bubeck, D. Molecular cell biology of complement membrane attack. Semin. Cell Dev. Biol. 72, 124-132 (2017).

2. Tomlinson, S., Taylor, P. W., Morgan, B. P. \& Luzio, J. P. Killing of gram negative bacteria by complement. Fractionation of cell membranes after complement C5b-9 deposition on to the surface of Salmonella minnesota Re595. Biochem. J. 263, 505-511 (1989).

3. Nakamura, M. et al. Quantification of the CD55 and CD59, membrane inhibitors of complement on HIV-1 particles as a function of complementmediated virolysis. Microbiol. Immunol. 40, 561-567 (1996).

4. Hoover, D. L., Berger, M., Nacy, C. A., Hockmeyer, W. T. \& Meltzer, M. S. Killing of Leishmania tropica amastigotes by factors in normal human serum. J. Immunol. 132, 893-897 (1984).

5. Harriman, G. R. et al. The role of C9 in complement-mediated killing of Neisseria. J. Immunol. 127, 2386-2390 (1981).

6. Nagata, M. et al. Inherited deficiency of ninth component of complement: an increased risk of meningococcal meningitis. J. Pediatr. 114, 260-264 (1989).

7. Meri, S. et al. Human protectin (CD59), an 18,000-20,000 MW complement lysis restricting factor, inhibits C5b-8 catalysed insertion of C9 into lipid bilayers. Immunology 71, 1-9 (1990).

8. Takeda, J. et al. Deficiency of the GPI anchor caused by a somatic mutation of the PIG-A gene in paroxysmal nocturnal hemoglobinuria. Cell 73, 703-711 (1993).

9. Taylor, R. P. et al. Hexamerization-enhanced CD20 antibody mediates complement-dependent cytotoxicity in serum genetically deficient in C9. Clin. Immunol. 181, 24-28 (2017). 
10. Podack, E. R. Molecular composition of the tubular structure of the membrane attack complex of complement. J. Biol. Chem. 259, 8641-8647 (1984).

11. Hadders, M. A. et al. Assembly and regulation of the membrane attack complex based on structures of C5b6 and sC5b9. Cell Rep. 1, 200-207 (2012).

12. DiScipio, R. G., Chakravarti, D. N., Muller-Eberhard, H. J. \& Fey, G. H. The structure of human complement component $\mathrm{C} 7$ and the C5b-7 complex. J. Biol. Chem. 263, 549-560 (1988).

13. Steckel, E. W., Welbaum, B. E. \& Sodetz, J. M. Evidence of direct insertion of terminal complement proteins into cell membrane bilayers during cytolysis. Labeling by a photosensitive membrane probe reveals a major role for the eighth and ninth components. J. Biol. Chem. 258, 4318-4324 (1983).

14. Podack, E. R., Tschoop, J. \& Müller-Eberhard, H. J. Molecular organization of C9 within the membrane attack complex of complement. Induction of circular C9 polymerization by the C5b-8 assembly. J. Exp. Med. 156, 268-282 (1982).

15. Serna, M., Giles, J. L., Morgan, B. P. \& Bubeck, D. Structural basis of complement membrane attack complex formation. Nat. Commun. 7, 10587 (2016).

16. Sharp, T. H., Koster, A. J. \& Gros, P. Heterogeneous MAC initiator and pore structures in a lipid bilayer by phase-plate cryo-electron tomography. Cell Rep. 15, 1-8 (2016).

17. Shatursky, O. et al. The mechanism of membrane insertion for a cholesteroldependent cytolysin: a novel paradigm for pore-forming toxins. Cell $\mathbf{9 9}$, 293-299 (1999).

18. Shepard, L. A. et al. Identification of a membrane-spanning domain of the thiol-activated pore-forming toxin Clostridium perfringens perfringolysin $\mathrm{O}$ : an alpha-helical to beta-sheet transition identified by fluorescence spectroscopy. Biochemistry 37, 14563-14574 (1998).

19. Rosado, C. J. et al. A common fold mediates vertebrate defense and bacterial attack. Science 317, 1548-1551 (2007).

20. Nguyen, T. H. D. et al. Cryo-EM structure of the yeast U4/U6.U5 tri-snRNP at $3.7 \AA ̊$ resolution. Nature 530, 298-302 (2016).

21. Lovelace, L. L., Cooper, C. L., Sodetz, J. M. \& Lebioda, L. Structure of human C8 protein provides mechanistic insight into membrane pore formation by complement. J. Biol. Chem. 286, 17585-17592 (2011).

22. Leung, C. et al. Real-time visualization of perforin nanopore assembly. Nat. Nanotechnol. 12, 467-473 (2017).

23. Sonnen, A. F., Plitzko, J. M. \& Gilbert, R. J. Incomplete pneumolysin oligomers form membrane pores. Open Biol. 4, 140044 (2014).

24. Dudkina, N. V. et al. Structure of the poly-C9 component of the complement membrane attack complex. Nat. Commun. 7, 10588 (2016).

25. Liu, T. et al. Human plasma N-glycoproteome analysis by immunoaffinity subtraction, hydrazide chemistry, and mass spectrometry. J. Proteome Res. 4, 2070-2080 (2005).

26. Franc, V., Yang, Y. \& Heck, A. J. Proteoform profile mapping of the human serum complement component C9 revealing unexpected new features of $\mathrm{N}$-, O-, and C-glycosylation. Anal. Chem. 89, 3483-3491 (2017).

27. Franc, V., Zhu, J. \& Heck, A. J. R. Comprehensive proteoform characterization of plasma complement component $\mathrm{C} 8 \alpha \beta \gamma$ by hybrid mass spectrometry approaches. J. Am. Soc. Mass Spectrom. 29, 1099-1110 (2018).

28. Lukoyanova, N. et al. Conformational changes during pore formation by the perforin-related protein pleurotolysin. PLoS Biol. 13, e1002049 (2015).

29. van Pee, K. et al. CryoEM structures of membrane pore and prepore complex reveal cytolytic mechanism of Pneumolysin. eLife 6, e23644 (2017).

30. Kremlitzka, M. et al. Functional analyses of rare genetic variants in complement component C9 identified in patients with age-related macular degeneration. Hum Mol Genet. 27, 2678-2688 (2018).

31. Bokori-Brown, M. et al. Cryo-EM structure of lysenin pore elucidates membrane insertion by an aerolysin family protein. Nat. Commun. 7, 11293 (2016).

32. Yoon, Y. Z., Hale, J. P., Petrov, P. G. \& Cicuta, P. Mechanical properties of ternary lipid membranes near a liquid-liquid phase separation boundary. J. Phys. Condens. Matter 22, 062101 (2010).

33. Bermúdez, H., Hammer, D. A. \& Discher, D. E. Effect of bilayer thickness on membrane bending rigidity. Langmuir 20, 540-543 (2004).

34. Elani, Y. et al. Measurements of the effect of membrane asymmetry on the mechanical properties of lipid bilayers. Chem. Commun. (Camb.) 51, 6976-6979 (2015).

35. de Meyer, F. J., Benjamini, A., Rodgers, J. M., Misteli, Y. \& Smit, B. Molecular simulation of the DMPC-cholesterol phase diagram. J. Phys. Chem. B 114, 10451-10461 (2010).

36. Silversmith, R. E. \& Nelsestuen, G. L. Interaction of complement proteins C5b-6 and C5b-7 with phospholipid vesicles: effects of phospholipid structural features. Biochemistry 25, 7717-7725 (1986).

37. Hu, V. W., Esser, A. F., Podack, E. R. \& Wisnieski, B. J. The membrane attack mechanism of complement: photolabeling reveals insertion of terminal proteins into target membrane. J. Immunol. 127, 380-386 (1981).

38. Esser, A. F., Kolb, W. P., Podack, E. R. \& Müller-Eberhard, H. J. Molecular reorganization of lipid bilayers by complement: a possible mechanism for membranolysis. Proc. Natl Acad. Sci. USA 76, 1410-1414 (1979).
39. Ruan, J., Xia, S., Liu, X., Lieberman, J. \& Wu, H. Cryo-EM structure of the gasdermin A3 membrane pore. Nature 557, 62-67 (2018).

40. Konovalova, A., Kahne, D. E. \& Silhavy, T. J. Outer membrane biogenesis. Annu. Rev. Microbiol. 71, 539-556 (2017).

41. Moskovich, O., Herzog, L. O., Ehrlich, M. \& Fishelson, Z. Caveolin-1 and dynamin-2 are essential for removal of the complement C5b-9 complex via endocytosis. J. Biol. Chem. 287, 19904-19915 (2012).

42. Sinha, B. et al. Cells respond to mechanical stress by rapid disassembly of caveolae. Cell 144, 402-413 (2011).

43. Romero, M. et al. Intrinsic repair protects cells from pore-forming toxins by microvesicle shedding. Cell Death Differ. 24, 798-808 (2017).

44. Giddings, K. S., Zhao, J., Sims, P. J. \& Tweten, R. K. Human CD59 is a receptor for the cholesterol-dependent cytolysin intermedilysin. Nat. Struct. Mol. Biol. 11, 1173-1178 (2004)

45. Johnson, S., Brooks, N. J., Smith, R. A., Lea, S. M. \& Bubeck, D. Structural basis for recognition of the pore-forming toxin intermedilysin by human complement receptor CD59. Cell Rep. 3, 1369-1377 (2013).

46. Husler, T., Lockert, D. H. \& Sims, P. J. Role of a disulfide-bonded peptide loop within human complement $\mathrm{C} 9$ in the species-selectivity of complement inhibitor CD59. Biochemistry 35, 3263-3269 (1996).

47. Lockert, D. H. et al. Identity of the segment of human complement C8 recognized by complement regulatory protein CD59. J. Biol. Chem. 270, 19723-19728 (1995)

48. Reister-Gottfried, E., Leitenberger, S. M. \& Seifert, U. Diffusing proteins on a fluctuating membrane: analytical theory and simulations. Phys. Rev. E 81, 031903 (2010).

49. Koch, M. et al. erythrocyte-binding antigen 175 triggers a biophysical change in the red blood cell that facilitates invasion. Proc. Natl Acad. Sci. USA 114, 4225-4230 (2017).

50. Marsh, D., Shanmugavadivu, B. \& Kleinschmidt, J. H. Membrane elastic fluctuations and the insertion and tilt of beta-barrel proteins. Biophys. J. 91, 227-232 (2006).

51. Zheng, S. Q. et al. MotionCor2: anisotropic correction of beam-induced motion for improved cryo-electron microscopy. Nat. Methods 14, 331-332 (2017).

52. Rohou, A. \& Grigorieff, N. CTFFIND4: fast and accurate defocus estimation from electron micrographs. J. Struct. Biol. 192, 216-221 (2015).

53. Fernandez-Leiro, R. \& Scheres, S. H. W. A pipeline approach to single-particle processing in RELION. Acta Crystallogr. D. Struct. Biol. 73, 496-502 (2017).

54. Pettersen, E. F. et al. UCSF Chimera--a visualization system for exploratory research and analysis. J. Comput. Chem. 25, 1605-1612 (2004).

55. Emsley, P., Lohkamp, B., Scott, W. G. \& Cowtan, K. Features and development of Coot. Acta Crystallogr. D. Biol. Crystallogr. 66, 486-501 (2010).

56. Webb, B. \& Sali, A. Protein structure modeling with MODELLER. Methods Mol. Biol. 1654, 39-54 (2017).

57. Phelan, M. M. et al. Solution structure of factor I-like modules from complement $\mathrm{C} 7$ reveals a pair of follistatin domains in compact pseudosymmetric arrangement. J. Biol. Chem. 284, 19637-19649 (2009).

58. Lopéz-Blanco, J. R. \& Chacón, P. iMODFIT: efficient and robust flexible fitting based on vibrational analysis in internal coordinates. J. Struct. Biol. 184, 261-270 (2013)

59. Topf, M. et al. Protein structure fitting and refinement guided by cryo-EM density. Structure 16, 295-307 (2008).

60. Pandurangan, A. P. \& Topf, M. RIBFIND: a web server for identifying rigid bodies in protein structures and to aid flexible fitting into cryo EM maps. Bioinformatics 28, 2391-2393 (2012).

61. Joseph, A. P. et al. Refinement of atomic models in high resolution EM reconstructions using Flex-EM and local assessment. Methods 100, 42-49 (2016).

62. Farabella, I. et al. a Python library for assessment of three-dimensional electron microscopy density fits. J. Appl. Crystallogr. 48, 1314-1323 (2015).

63. Afonine, P. V. et al. Real-space refinement in PHENIX for cryo-EM and crystallography. Acta Crystallogr. D. Struct. Biol. 74, 531-544 (2018).

64. Krivov, G. G., Shapovalov, M. V. \& Dunbrack, R. L. Improved prediction of protein side-chain conformations with SCWRL4. Proteins 77, 778-795 (2009).

65. Williams, C. J. et al. MolProbity: more and better reference data for improved all-atom structure validation. Protein Sci. 27, 293-315 (2018).

66. Purushothaman, S., Cicuta, P., Ces, O. \& Brooks, N. J. Influence of high pressure on the bending rigidity of model membranes. J. Phys. Chem. B 119, 9805-9810 (2015).

\section{Acknowledgements}

We thank A. Carter for discussions; D. Clare, S. Welch, A. Seibert, C. Hecksel, and F. de Haas for data acquisition assistance; S. Islam for computational support, and J. Lo for help in selection of particles. We thank the LMB-MRC for access to electron microscopes and Diamond Light Source for access to eBIC (proposals EM19429, EM13304, EM16919, EM13893, EM12204, and EM12388) funded by the Wellcome Trust, MRC and BBSRC This work is supported by a CRUK Career Establishment Award (C26409/A16099) to D.B.; C.M.B. is funded by a BBSRC Doctoral Training Program grant, Ref: BB/J014575/1; 
N.J.B. is supported by a EPSRC Programme Grant (EP/ J017566/1); A.P.J. and M.T. are supported by MRC (MR/M019292/1).

\section{Author contributions}

M.S. and A.M. prepared samples. M.S., A.M., C.M.B., and D.B. performed the electron microscopy. M.S., A.M., D.B., C.M.B., and S.G. contributed to the image processing. M.S., C.M.B., A.P.J., D.B., and M.T. performed molecular modeling. A.M. and N.J.B. performed flicker spectroscopy. B.P.M. contributed to discussions and analysis of MAC structure. All authors contributed to the experimental design, data analysis, and preparation of the manuscript. M.S. and C.M.B. contributed equally.

\section{Additional information}

Supplementary Information accompanies this paper at https://doi.org/10.1038/s41467018-07653-5.

Competing interests: The authors declare no competing interests.

Reprints and permission information is available online at http://npg.nature.com/ reprintsandpermissions/

Publisher's note: Springer Nature remains neutral with regard to jurisdictional claims in published maps and institutional affiliations.

\section{(c) (i)}

Open Access This article is licensed under a Creative Commons Attribution 4.0 International License, which permits use, sharing, adaptation, distribution and reproduction in any medium or format, as long as you give appropriate credit to the original author(s) and the source, provide a link to the Creative Commons license, and indicate if changes were made. The images or other third party material in this article are included in the article's Creative Commons license, unless indicated otherwise in a credit line to the material. If material is not included in the article's Creative Commons license and your intended use is not permitted by statutory regulation or exceeds the permitted use, you will need to obtain permission directly from the copyright holder. To view a copy of this license, visit http://creativecommons.org/ licenses/by/4.0/.

(c) The Author(s) 2018 